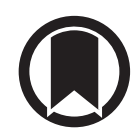

CrossMark

\section{Responding to SARS-CoV-2 in South Africa: what can we learn from drug- resistant tuberculosis?}

\author{
To the Editors:
}

The novel coronavirus strain, severe acute respiratory syndrome coronavirus 2 (SARS-CoV-2), was first reported from China in December 2019 [1]. As of 14 May, 2020, more than 4.4 million individuals have tested positive for SARS-COV-2 globally [2]. More than 300000 individuals have died globally due to SARS-COV-2 [2]. In South Africa, cumulatively at the same time point, 12739 individuals have been infected and 238 deaths reported [2]. Tuberculosis (TB) is the leading infectious disease cause of death with 1.4 million deaths in 2018 [3]. Drug-resistant tuberculosis (DR-TB) is a threat to TB control globally. Over the past decade, several interventions have improved the outcomes of DR-TB patients and reduced the burden of disease. We discuss lessons from DR-TB interventions in South Africa that could be helpful in the fight against SARS-COV-2.

1) Rapid adoption of new diagnostics: Early awareness, testing and diagnosis are important factors in successful management. The use of GeneXpert MTB/RIF brought about a revolution in diagnosing DR-TB reducing time to detection from weeks to days. The recommended primary diagnostic for SARS-COV-2 detection is molecular using the PCR method. Several rapid molecular diagnostic methods are available and should be rapidly evaluated and implemented. South Africa has adopted several and recently also introduced the GeneXpert Xpress SARS-CoV-2 cartridge diagnosing coronavirus disease 2019 (COVID-19) within $45 \mathrm{~min}$, leveraging the GeneXpert platform that is decentralised and extensively used for TB diagnosis.

2) Parallel process of research and implementation: The Bedaquiline Clinical Access Programme (BCAP) provided access to a novel drug for DR-TB patients, generated local evidence to support decision-making and led to rapid adoption, bolstering the fight against an infectious disease [4]. We managed to reduce mortality among DR-TB patients after updating our clinical guidelines using local data (figure 1) [5]. Death rate decreased from 45\% (2010 cohort) to 21\% (2016 cohort) among extensively drug-resistant TB patients and treatment success rate improved from $9 \%$ to $58 \%$ during the same period in this group. Importantly, outcomes were monitored closely after introduction of novel regimens and ensured that data is put in the public domain in a timely manner. The BCAP model is a good example and we encourage rapid referral to COVID-19 clinical trials being conducted; the Solidarity clinical trial is an example and will evaluate new and repurposed agents to treat COVID-19. Another example of a research project that helped drive development of country-specific policies is the surveillance of adverse events conducted by BoRisov et al. [6].

3) Decentralisation of complex services for easy access for community members: Bringing screening, testing and treatment close to where people live has proved to produce better treatment outcomes for DR-TB patients in South Africa and helped reduce the cost of DR-TB care [7]. Such interventions are key to success in the fight against SARS-COV-2. Effective decentralisation facilitates contact tracing important for both conditions and has been well resourced for COVID-19. It is a missed opportunity, however, not to test for TB when investigating COVID-19, and vice versa. This is recommended by the World Health Organization: local adoption is now planned and should be considered in high burden TB settings.

@ERSpublications

Rapid adoption of new diagnostic tools, parallel process of research and implementation, decentralisation of services, the use of personal protective equipment, as well as strong partnership and collaboration, could strengthen the fight against COVID-19 https://bit.ly/2XupDNe

Cite this article as: Ndjeka N, Conradie F, Meintjes G, et al. Responding to SARS-CoV-2 in South Africa: what can we learn from drug-resistant tuberculosis?. Eur Respir J 2020; 56: 2001369 [https://doi.org/ $10.1183 / 13993003.01369-2020]$. 

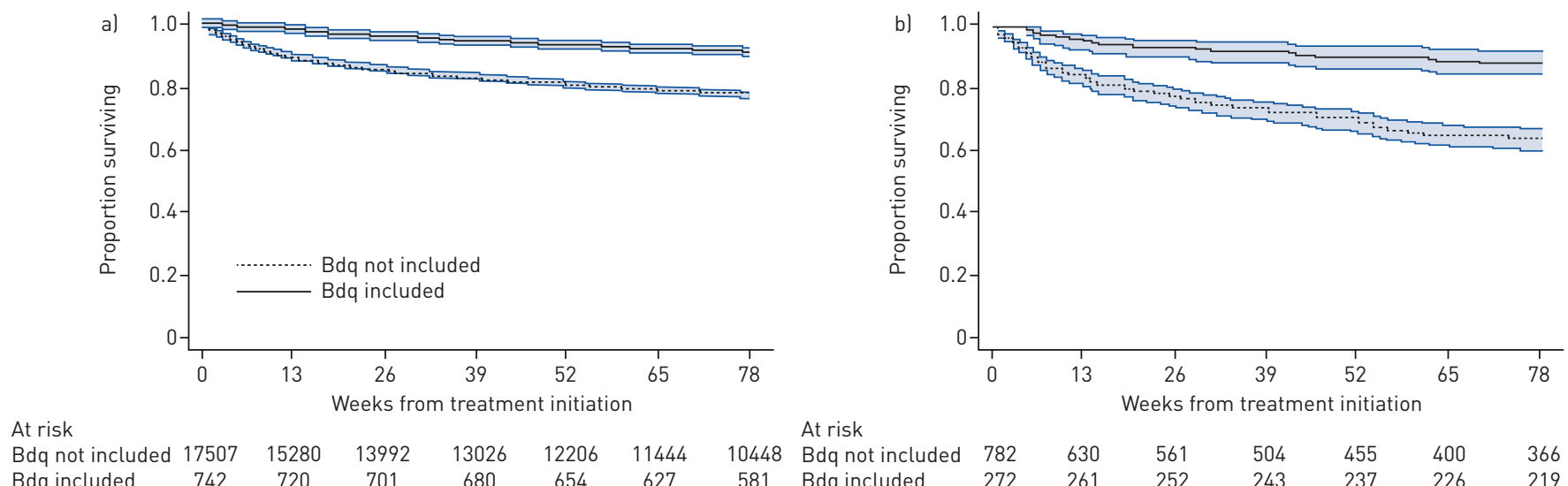

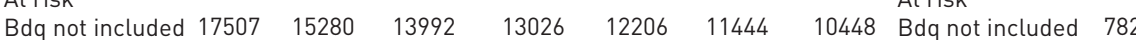

At risk

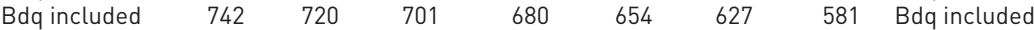

$\begin{array}{lllllll}782 & 630 & 561 & 504 & 455 & 400 & 366 \\ 272 & 261 & 252 & 243 & 237 & 226 & 219\end{array}$

FIGURE 1 Kaplan-Meier survival curves for a) multidrug/rifampicin-resistant tuberculosis and b) extensively drug-resistant tuberculosis, by regimen inclusive of bedaquiline (Bdq), with 95\% confidence intervals, for patients started on treatment between 1 July, 2014 and 31 March, 2016. Reproduced with permission from the publisher [5].

4) The use of personal protective equipment (PPE) by healthcare workers and patients:

The use of PPE, especially in clinical environments, has been associated with reduction of DR-TB transmission among healthcare workers in various settings. Administrative and environmental factors are also critically important for DR-TB. The major challenge has been scarcity of PPE in various areas where they are needed to manage unsuspected, undiagnosed infectious patients and this is being adequately addressed.

5) Strong partnership and collaboration: This is central to the response and led to improvement of DR-TB treatment outcomes. Government officials, academia, non-governmental organisations and civil society worked together in coalition, driving the successes observed [8].

6) Management of TB and COVID-19 co-infection: as both conditions affect the respiratory system with overlapping symptoms, clinicians are urged to test for both. This is particularly important to prevent nosocomial spread when hospitalisation is needed for either. A total of 776 beds have been identified out of 3798 beds at 33 DR-TB treatment sites. Although the first TB-COVID-19 cohorts reported did not show a high death rate in this group [9, 10], HIV co-infection among TB is 59\% in South Africa and is an additional risk. Integrating COVID-19 activities with TB (and HIV) programmes is important.

In conclusion, the fight against SARS-COV-2 can be won if we continue to make effective use of rapid diagnostic tools and effective contact tracing, decentralise treatment services, and strengthen the use of personal protective devices in areas that need them most. Equally importantly, partnership between governments and the private sector, political commitment and learning implementation may go a long way to defeating SARS-COV-2.

Norbert Ndjeka ${ }^{1,13}$, Francesca Conradie ${ }^{2,13}$, Graeme Meintjes ${ }^{3}$, Anja Reuter ${ }^{4}$, Jennifer Hughes $\oplus^{5}$, Xavier Padanilam ${ }^{6}$, Nazir Ismail ${ }^{7}$, Yulene Kock $^{1}$, Iqbal Master ${ }^{8}{ }^{8}$, Rodolfo Romero ${ }^{9}$, Julian te Riele ${ }^{10}$, Martin Enwerem ${ }^{11}$, Hannetjie Ferreira ${ }^{12}$ and Gary Maartens ${ }^{3}$

${ }^{1}$ National Dept of Health, Drug Resistant TB Directorate, Pretoria, South Africa. ${ }^{2}$ University of Witwatersrand, Faculty of Health Sciences, Dept of Clinical Medicine, Johannesburg, South Africa. ${ }^{3}$ Dept of Medicine and Wellcome Centre for Infectious Diseases Research in Africa, Institute of Infectious Disease and Molecular Medicine, University of Cape Town, Rondebosch, South Africa. ${ }^{4}$ Médecins Sans Frontières, Khayelitsha, South Africa. ${ }^{5}$ Desmond Tutu TB Centre, Dept of Paediatrics and Child Health, Faculty of Medicine and Health Sciences, Stellenbosch University, Cape Town, South Africa. ${ }^{6}$ Sizwe Tropical Disease Hospital, Dept of Health, Gauteng, South Africa. ${ }^{7}$ Centre for Tuberculosis, National Institute for Communicable Diseases, Johannesburg, South Africa. ${ }^{8}$ King Dinuzulu Hospital, Kwazulu Natal Provincial Dept of Health, Durban, South Africa. ${ }^{9}$ District Clinical Specialist Team, Namakwa, South Africa. ${ }^{10}$ Metro TB Hospital Centre, Brooklyn Chest Hospital, Cape Town, South Africa. ${ }^{11}$ Witbank TB Specialized Hospital, Witbank, South Africa. ${ }^{12}$ Klerksdorp-Tshepong Hospital, Klerksdorp, South Africa. ${ }^{13}$ Joint first author: N. Ndjeka and F. Conradie contributed equally.

Correspondence: Norbert Ndjeka, National TB Programme, Republic of South Africa, P/bag X828 Pretoria, 0001, South Africa. E-mail: Norbert.Ndjeka@health.gov.za 
Received: 23 April 2020 | Accepted after revision: 21 May 2020

Conflict of interest: None declared.

\section{References}

1 World Health Organization (WHO). Rolling Updates on Coronavirus Disease (COVID-19). www.who.int/ emergencies/diseases/novel-coronavirus-2019/events-as-they-happen Date last accessed: 20 April 2020.

2 Johns Hopkins University. Johns Hopkins Coronavirus Dashboard. Track the Coronavirus Outbreak on Johns Hopkins Live Dashboard. https://coronavirus.jhu.edu/map.html Date last accessed: 14 May 2020.

3 World Health Organization (WHO). Global TB Report. Geneva, WHO, 2019. Available from: www.who.int/tb/ global-report-2019

4 Ndjeka N, Schnippel K, Master I, et al. High treatment success rate for multidrug-resistant and extensively drug-resistant tuberculosis using a bedaquiline-containing treatment regimen. Eur Respir J 2018; 52: 1801528.

5 Schnippel K, Ndjeka NO, Maartens G, et al. Effect of bedaquiline on mortality in South African patients with drug-resistant tuberculosis: a retrospective cohort study. Lancet Respir Med 2018; 6: 699-706.

6 Borisov S, Danila E, Maryandyshev A, et al. Surveillance of adverse events in the treatment events in the treatment of drug-resistant tuberculosis: first global report. Eur Respir J 2019; 54: 1901522.

7 Sinanovic E, Ramma L, Vassall A, et al. Impact of reduced hospitalisation on the cost of treatment for drug-resistant tuberculosis in South Africa. Int J Tuberc Lung Dis 2015; 19: 172-178.

8 Conradie F, Meintjes G, Hughes J, et al. Clinical Access Program for Bedaquiline for the treatment of drug-resistant tuberculosis. S Afr Med J 2014; 104: 164-166.

9 Tadolini M, Codecasa LR, Garcia-Garcia JM, et al. Active tuberculosis, sequelae and COVID-19 co-infection: first cohort of 49 cases. Eur Respir J 2020; 56: 2001398.

10 Motta I, Centis R, D'Ambrosio L, et al. Tuberculosis, COVID-19 and migrants: preliminary analysis of deaths occurring in 69 patients from two cohorts. Pulmonology 2020; 26: 233-240. 